\title{
Procrastinación, ansiedad ante los exámenes y rendimiento académico en estudiantes universitarios
}

\author{
Procrastination, test anxiety and academic performance
}

on university students

\author{
Malena Manchado Porras ${ }^{1}$ y Federico Hervías Ortega ${ }^{2}$ \\ ${ }^{1}$ Graduada en Psicología. Personal Investigador en Formación del Departamento de Psicología, \\ Facultad de Ciencias de la Educación, Universidad de Cádiz, España. \\ E-mail: malena.manchadoporras@alum.uca.es \\ ${ }^{2}$ Doctor en Psicología. Profesor Contratado Doctor del Área de Personalidad, Evaluación y \\ Tratamiento Psicológico. Departamento de Psicología, Facultad de Ciencias de la Educación, \\ Universidad de Cádiz. E-mail: federico.hervias@uca.es
}

Facultad de Ciencias de la Educación, Universidad de Cádiz. Cádiz, España.

\section{Resumen}

La procrastinación se define como una tendencia conductual a postergar tareas que, por sus características poco motivadoras o tediosas, no son atractivas para quien las realiza, y cuyo retraso puede acarrear consecuencias negativas. El propósito de este estudio es examinar el efecto de la procrastinación académica y la ansiedad ante los exámenes sobre el rendimiento académico, así como la relación entre ambas variables. Para ello, se ha realizado una investigación con 201 estudiantes universitarios empleando el Inventario Alemán de Ansiedad frente a los Exámenes Adaptado (GTAI-A), la Escala de Procrastinación Académica (EPA) y un registro de las calificaciones obtenidas por los participantes recogidos a través de un autoinforme. Los resultados revelan la presencia de ansiedad ante la evaluación y la procrastinación académica en estudiantes universitarios, e indican que la ansiedad ante los exámenes muestra una relación negativa con el rendimiento académico. De igual forma, la procrastinación se relaciona con dos factores de ansiedad (interferencia y falta de confianza), incidiendo de forma indirecta en dicho rendimiento. Adicionalmente, se han encontrado diferencias significativas en el rendimiento académico en función de los diferentes niveles de ansiedad identificados $\left(\chi_{(2,201)}^{2}=16.929 ; p\right.$ $=.001)$. En conclusión, para prevenir e intervenir en el bajo rendimiento académico y en los problemas psicosociales asociados, los servicios universitarios de atención psicológica deben considerar la influencia de múltiples variables, de forma que se apliquen programas de intervención específicos para cada dimensión, aumentando así los niveles de eficacia y eficiencia en su labor asistencial. Palabras clave: ansiedad ante los exámenes, procrastinación, rendimiento académico, estudiantes universitarios.

\begin{abstract}
Procrastination is defined as a behavioral tendency to postpone tasks that, because of their unmotivating or tedious characteristics, are not attractive to the performer, and whose delay can lead to negative consequences. This
\end{abstract}


is the behavior of delaying responsibilities, being aware of their importance and the repercussions of the delay. These consequences can affect the personal, social, occupational and academic spheres of individuals. Therefore, the purpose of this study is to examine the relationship of academic procrastination and test anxiety on academic performance, as well as the relationship between the two variables. In this way, the aim is to achieve a better understanding of the links between the three variables, which will serve as a theoretical basis for preventing and intervening in the negative repercussions of procrastination. To this end, an investigation has been carried out with 201 college students, using the German Test Anxiety Inventory (GTAI-A), the Academic Procrastination Scale (EPA), and a record of the qualifications obtained by the participants collected through a self-report. This is a correlational design that does not include experimental manipulation of variables or randomization of the sample, so the aim is not to establish causal relationships but to determine the direction and strength of the associations between variables. The results reveal the presence of academic procrastination and test anxiety in university students. Specifically, the majority of participants (45.3 $\%$ ) reflected a medium level of anxiety, 28.9 $\%$ reflected a high level and $25.9 \%$ showed a low level. In the total sample, procrastination showed a mean score of 30.3 points out of 60 . As for the relationships of both variables with academic performance, test anxiety shows a negative relationship with academic performance, so higher levels of anxiety are associated with worse grades. Procrastination does not show a significant and direct relationship with grades, but it is related to two anxiety factors (interference and lack of confidence), indirectly affecting academic performance. In addition, significant differences in academic performance have been found based on the different anxiety levels identified $\left(\chi_{(2,201)}^{2}=16.929 ; p=.001\right)$, with the high anxiety group obtaining a lower mean score $(M=5.74 ; S D=1.27)$. This supports the hypothesis that test anxiety is negatively associated with academic performance. Moreover, it has been found that comparing the medium and low anxiety groups, the differences are not significant, but comparing each of them with the high anxiety group they are. Therefore, significant differences in the grades only appear when the level of anxiety is high. That is, a moderate level of anxiety is adaptive, and only becomes detrimental if it becomes high. In conclusion, to prevent and intervene in academic underachievement and associated psychosocial problems, university psychological services should consider the influence of test anxiety, procrastination and other cognitive, behavioral, motivational and emotional variables. According to this study, intervention in test anxiety is a central aspect, as it has a direct effect on academic performance. In the same way, procrastinating behaviors can be intervened, as they interfere indirectly in academic outcomes, being associated with cognitive manifestations of test anxiety. The relative weight of the different variables associated with academic achievement varies according to each student, so that an individualized approach to these types of problems would increase the levels of effectiveness and efficiency in the care provided by university psychological services. Finally, the need for more research to identify the factors associated with low academic performance and the relationships between them is highlighted, in order to facilitate the design of effective interventions for different student profiles.

Keywords: test anxiety, procrastination, academic performance, university students.

\section{Introducción}

La relación entre la procrastinación y las distintas consecuencias académico-laborales, sociales y psicológicas está generando un creciente interés en el ámbito de la psicología (Moreta y Durán, 2018). En la esfera académica, la conducta de procrastinación se 
vincula con la ansiedad ante los exámenes, ya sea como antecedente o como consecuente. Por ello, estudiar ambos constructos y la relación entre ellos es esencial para abordar las posibles repercusiones en el ámbito académico y personal (Pardo, Perilla y Salinas, 2016).

El término "procrastinación" hace referencia a la demora o el retraso de responsabilidades personales que tienen plazos temporales preestablecidos, a pesar de ser consciente de la importancia de esas responsabilidades y de las repercusiones negativas de retrasarlas (Lay, 1990). En este sentido, Steel (2007) asume que procrastinar consiste en retrasar voluntariamente una tarea a pesar de saber que ese retraso va a producir malestar. En relación con las causas de la procrastinación, la concepción actual del fenómeno las sitúa en distintos factores, entre los que destacan las expectativas de resultados, la valoración del sujeto sobre la relevancia de la tarea, el tiempo necesario para obtener gratificación y el grado de impulsividad del individuo (Angarita, 2012).

Desde una visión conductual, la procrastinación se produce y se mantiene por reforzamiento. Concretamente, se da porque ha tenido consecuencias positivas a corto plazo, en lugar de resultados positivos a largo plazo (Carranza y Ramírez, 2013). Desde una visión cognitivo-conductual, la explicación de la procrastinación se encontraría en los pensamientos irracionales sobre las implicaciones de terminar la tarea de forma óptima. Así, el modelo cognitivo de Wolters (2003) propone que la persona que procrastina cuenta con esquemas desadaptativos sobre su baja autoeficacia frente a las tareas. Esos esquemas desadaptativos son patrones cognitivos que provocan que la persona, ante una tarea o responsabilidad, experimente pensamientos automáticos sobre su incapacidad para planificarla o llevarla a cabo. De la misma forma, Contreras et al. (2005) señalan que los pensamientos negativos sobre la escasa capacidad para finalizar con éxito las tareas llevan a la persona a realizar conductas de procrastinación.
La procrastinación se manifiesta en diversas conductas, en función de la situación o el contexto específico en la que se aplazan las tareas. Según Steel (2007), la procrastinación puede presentarse en el contexto laboral, en la política, en el ámbito bancario, en la educación, etcétera. De esta forma, una persona puede aplazar distintas tareas y responsabilidades, en función del ámbito concreto en el que se presente la procrastinación. Por tanto, el fenómeno se extiende a una gran diversidad de contextos, incluido el mantenimiento de la salud, el ámbito familiar o de pareja, e incluso el ocio.

Finalmente, se pueden diferenciar dos tipos de personas procrastinadoras: las activas y las pasivas (Hsin y Nam, 2005, citado por Alegre, 2013). Estas últimas se ajustan a la concepción tradicional de la procrastinación, ya que aplazan las tareas a pesar de las consecuencias negativas que conlleva ese aplazamiento. Por el contrario, los procrastinadores activos realizan esa postergación de forma voluntaria, ya que prefieren trabajar bajo presión. De esta forma, los procrastinadores activos muestran grandes diferencias con los pasivos, que incluyen la percepción de autoeficacia, el uso de estrategias de afrontamiento adaptativas y la adecuada gestión del tiempo. Además, habitualmente este tipo de procrastinación no conlleva las consecuencias negativas que implica la procrastinación pasiva, lo cual le otorga al fenómeno una connotación positiva en lugar de negativa (Alegre, 2016).

Concretamente, la procrastinación académica supone un retraso en el inicio de las tareas académicas, tales como la realización de actividades con un plazo preestablecido o el estudio del contenido de los exámenes con suficiente antelación. Además, normalmente las actividades académicas se postergan para sustituirlas por actividades sociales o lúdicas, más gratificantes a corto plazo (Díaz-Morales, 2019). La procrastinación académica puede asociarse con un bajo nivel de autocontrol, ya que este se concibe como la capacidad de elegir conductas que conllevan consecuen- 
cias más valiosas a largo plazo, en lugar de conductas con consecuencias más inmediatas, pero menos valiosas. Adicionalmente, se puede relacionar con un bajo nivel de autoeficacia. Es decir, si la persona considera que no cuenta con las habilidades requeridas para ejecutar una tarea y obtener éxito, será más probable que postergue esa tarea con el fin de evitar que se manifieste ese déficit de habilidades (Quant y Sánchez, 2012).

La ansiedad ante la evaluación es otro elemento que se relaciona de forma significativa con la procrastinación académica. Según Furlan, Heredia, Piemontesi y Tuckman (2012), existe una correlación positiva entre la ansiedad ante los exámenes y la procrastinación. Concretamente, la procrastinación se relaciona de forma positiva y significativa con las dimensiones de ansiedad, interferencia y falta de confianza. En este sentido, las personas que procrastinan revelan un mayor grado de ansiedad en momentos próximos a la fecha de evaluación, ya que son conscientes del escaso tiempo disponible para preparar la prueba (Marquina, Gómez, Salas, Santibañez y Rumiche, 2016). Sin embargo, la ansiedad ante los exámenes no supone un problema clínicamente significativo a menos que interfiera en el funcionamiento habitual de un individuo dificultando la ejecución de los exámenes, provocando su evitación o generando malestar en la persona. De esta forma, si aparece en niveles moderados puede ayudar al estudiante a anticipar una futura situación de evaluación y a prepararse para prevenir las posibles consecuencias negativas (Furlan, Ferrero y Gallart, 2014).

Finalmente, el bajo rendimiento académico es otro fenómeno asociado a la procrastinación. El rendimiento académico puede entenderse como el logro de los objetivos preestablecidos de una asignatura, que se manifiesta mediante las calificaciones obtenidas en una prueba de evaluación (Caballero, Abello y Palacio, 2007, citado por Lamas, 2015). Las variables que repercuten en los resultados académicos son diversas: la capacidad intelectual, la personalidad, la autoestima, la motivación, las rutinas de estudio, la relación entre el profesor y el alumno, el contexto familiar, socioeconómico y cultural, entre otras (Chilca, 2017; Gómez, Oviedo y Martínez, 2011). Así, como se refleja en el trabajo de Furlan et al. (2012), la procrastinación es una de esas variables, la cual refleja una relación negativa con el rendimiento académico. Además, según el trabajo de Cerda, Saiz y Vergara (2018), existe una relación positiva entre tenacidad y desempeño académico. Es decir, aquellos estudiantes más tenaces, situados en el quintil cinco de tenacidad, superaron significativamente al resto de los participantes en el promedio de calificaciones académicas. Esto podría relacionarse con los elementos causales de la procrastinación resaltados por Angarita (2012) y citados al inicio de este apartado. Es decir, aquellos estudiantes más impulsivos y con menor capacidad para aplazar la gratificación tenderán a procrastinar. En consecuencia, la procrastinación repercutirá de manera negativa en sus resultados académicos, ya que el logro de un óptimo rendimiento académico requiere planificación, automonitorización y persistencia, especialmente cuando la tarea es compleja y altamente estructurada (Credé, 2016; citado por Cerda et al., 2018).

En referencia a la conexión entre el rendimiento académico y la ansiedad ante la evaluación, existen investigaciones que indican una relación inversa entre ambos elementos (Dordi Nejad, et al., 2011; Rana y Mahmood, 2010). Una de esas investigaciones es el estudio realizado por Piemontesi y Heredia (2011), en el cual se indica que existe una relación negativa entre el promedio de calificaciones autoinformado y la ansiedad ante los exámenes. Además, dicho estudio también añade que las dimensiones de ansiedad que se relacionan con el promedio autoinformado de calificaciones son la interferencia y la falta de confianza, ambas de forma negativa. Sin embargo, Farooqui, Ghani y Spielberger (2012) han encontrado una relación débil entre rendimiento académico y ansiedad, y otras 
investigaciones como la de Álvarez, Aguilar y Lorenzo (2012) han revelado incluso una falta de asociación entre ambas variables.

En consecuencia, a pesar de la asociación de la procrastinación con la ansiedad ante la evaluación y con el bajo rendimiento académico, el aplazamiento de actividades académicas no conlleva siempre la experiencia de ansiedad (Sánchez Hernández, 2010). En ocasiones, la conducta procrastinadora desemboca en resultados académicos positivos, por lo que dicha conducta se ve reforzada. Es decir, es posible obtener un buen rendimiento a pesar de llevar a cabo conductas de procrastinación. De esta forma, los individuos generan la creencia de que rinden mejor bajo un estado de presión.

Acerca de la procrastinación académica, la literatura indica que se trata de un fenómeno aún más prevalente que la procrastinación general, con una incidencia en los estudiantes de entre el $30 \%$ y el $60 \%$ (González-Brignardello y Sánchez-Elvira-Paniagua, 2013). Así, el estudio de Balkis y Duru (2009) reveló que el $23 \%$ de los estudiantes que participaron presentaba un nivel alto de procrastinación y el $27 \%$ presentaba un nivel moderado. En relación con la ansiedad ante los exámenes, también se trata de un fenómeno frecuente en la población estudiantil. Como problema clínico, el estudio de Furlan, Pérez, Moyano y Cassady (2011) señala una prevalencia en torno al $10 \%$. Por su lado, el estudio de Domínguez, Bonifacio y Caro (2016) revela cifras incluso superiores, y encuentra una elevada ansiedad ante los exámenes en el $26.23 \%$ de los estudiantes.

Desde los servicios de atención psicológica universitarios se trabaja intensamente para paliar las consecuencias negativas de aquellos comportamientos asociados a estados de ansiedad académica, que además se relacionan con conductas procrastinadoras (Saúl, López-González y Bermejo, 2009; Marchena et al., 2009). Además de realizarse sesiones de psicoterapia individualizada para atender los casos clínicos y subclínicos (Hervías,
Marchena y Menacho, 2020), estos servicios desarrollan su actividad implementando cursos, talleres y seminarios dirigidos al aprendizaje de competencias académicas y personales de tipo procedimental, como cursos de organización del tiempo, técnicas de memorización, desarrollo de textos científicos, trabajo en equipo, hablar en público, gestión de la ansiedad ante los exámenes, motivación, gestión del estrés y técnicas de relajación, etcétera (Marchena et al., 2011).

De esta manera, el objetivo principal de este trabajo es comprobar la relación de la procrastinación académica y la ansiedad con los exámenes en el rendimiento académico, asumiendo que los resultados académicos están determinados por múltiples variables además de las citadas. De forma específica, se trata de examinar los niveles de ansiedad ante los exámenes y los niveles de procrastinación académica en población universitaria, examinar la relación de cada una de esas variables con el rendimiento académico y la relación de ambas variables entre sí. Así, se establecen como hipótesis de trabajo que tanto la ansiedad académica como la procrastinación son fenómenos existentes en la población universitaria; ambos fenómenos tendrán una relación positiva entre ellos y se relacionarán de forma negativa con el rendimiento académico.

\section{Método}

\section{Participantes}

Los integrantes del estudio se seleccionaron mediante un muestreo no probabilístico por conveniencia. Es decir, el único criterio de selección fue ser estudiante universitario, por lo que se eligió una muestra de fácil acceso formada por los alumnos de aquellos docentes universitarios que permitieron la administración de las pruebas en su horario de clase. Por tanto, las respuestas de los participantes fueron registradas en un contexto académico, con el fin de conseguir de forma eficaz información 
sobre un número considerable de estudiantes universitarios. Finalmente, se alcanzó un total de 201 participantes, de los cuales 164 eran mujeres y 37 eran hombres. Esta diferencia entre los tamaños de ambos grupos se debe fundamentalmente a los centros y titulaciones que participaron en este estudio (grados en Educación infantil y Psicología) con una fuerte presencia de mujeres sobre hombres.

\section{Instrumentos}

Los datos de la investigación se registraron de dos modos diferentes. Por un lado, se emplearon dos tests psicométricos. Por otro, se realizó un registro de las calificaciones obtenidas por los participantes tras la realización de pruebas de evaluación académica.

\section{Inventario Alemán de Ansiedad frente a los Exámenes Adaptado (GTAI-A)}

Se trata de una adaptación del German Test Anxiety Inventory (TAI-G) (Hodapp, 1991) realizada por Heredia, Piemontesi, Furlan y Volker (2008). El test está compuesto por 28 ítems que conforman cuatro escalas: Preocupación, Emocionalidad, Interferencia y Falta de Confianza. En primer lugar, la preocupación hace referencia a los pensamientos sobre las repercusiones negativas del fracaso. En segundo lugar, la emocionalidad alude a la percepción subjetiva de respuestas fisiológicas y de las emociones provocadas por esas respuestas. Por su lado, la interferencia se refiere a los pensamientos intrusivos o irrelevantes que impiden focalizar la atención en la tarea. Para terminar, la falta de confianza tiene un sentido inverso a lo que su denominación indica: hace referencia a la seguridad en la capacidad de uno mismo para realizar la tarea (Montaña, 2011). La escala total y las cuatro subescalas revelaron índices de confiabilidad apropiados, con coeficientes alfa de Cronbach entre .74 y .90 (Piemontesi, Heredia y Furlan, 2012). Los reactivos son ítems de tipo Likert, con cuatro alternativas de respuesta (nunca, casi nunca, casi siempre y siempre).

\section{Escala de Procrastinación Académica (EPA; Busko, 1998)}

La versión original del instrumento fue adaptada al español por Álvarez-Blas (2010) y revisada más tarde en el estudio de Domínguez, Villegas y Centeno (2014). A partir de dicho estudio, se obtuvo un instrumento final de 12 ítems. El instrumento presenta una estructura bifactorial, y revela un índice de confiabilidad apropiado (alfa de Cronbach de .816 para la escala total; .821 para el factor autorregulación académica y .752 para el factor postergación de actividades). Los reactivos de la versión final de la escala son de tipo Likert, con cinco alternativas de respuesta (nunca, casi nunca, a veces, casi siempre y siempre) y presentan una estructura bifactorial. La confiabilidad se estimó mediante el alfa de Cronbach y se obtuvo .816 para la escala total, .821 para el factor autorregulación académica y .752 para el factor postergación de actividades.

\section{Registro de las calificaciones}

Se llevó a cabo mediante una hoja de anotación a completar por los participantes, en la que cada individuo indicó el número de asignaturas en las que se había matriculado en un primer semestre de un curso académico, el número de asignaturas a cuya evaluación se había presentado en la primera convocatoria de exámenes y el número de asignaturas aprobadas en dicha convocatoria. Además, cada sujeto informó la nota obtenida en cada una de las asignaturas de las que se examinó en la convocatoria en cuestión (en una escala de 0 a 10), incluidas las asignaturas suspensas, de forma que se pudo obtener un promedio académico autodeclarado por parte de los participantes.

\section{Diseño}

El presente estudio, de tipo ex post facto retrospectivo de grupo simple, es una inves- 
tigación correlacional que no cuenta con manipulación experimental de variables ni aleatorización de la muestra. Por lo tanto, no busca establecer relaciones causales inequívocas, sino constatar la dirección y la fuerza de distintas asociaciones entre variables.

\section{Procedimiento}

Los datos de los participantes se registraron mediante los instrumentos mencionados con anterioridad. Las dos pruebas estandarizadas se administraron durante las dos semanas previas al inicio de un período de exámenes en un curso académico, para registrar así los niveles de procrastinación y de ansiedad ante los exámenes existentes en los participantes justo antes de comenzar un período de esfuerzo y trabajo intenso. Posteriormente, una vez concluido el período de exámenes, se llevó a cabo la segunda recogida de datos, en este caso relativos a las calificaciones obtenidas. De este modo, se pudo obtener una calificación media en dicha convocatoria por cada miembro de la muestra. La calificación media se tomó como indicador del rendimiento académico.

\section{Resultados}

En la Tabla 1 se pueden observar algunos datos descriptivos relativos a las variables centrales de la investigación. Para una mejor comprensión de los datos indicados en la tabla se debe aclarar que la puntuación máxima es de 84 puntos en el cuestionario de ansiedad ante los exámenes y de 60 puntos en el cuestionario de procrastinación académica. Además, la calificación media puede encontrarse en un máximo de 10 puntos.

Tabla 1

Estadísticos descriptivos.

\begin{tabular}{|l|l|l|l|l|l|}
\hline & $\mathrm{N}$ & Mín. & Máx. & $D T$ & $M$ \\
\hline Calificación media & 201 & 3 & 9 & 1.29 & 6.28 \\
Ansiedad ante los exámenes & 201 & 22 & 73 & 10.79 & 48.52 \\
Procrastinación académica & 201 & 14 & 53 & 7.07 & 30.30 \\
N válido (según lista) & 201 & & & & \\
\hline
\end{tabular}

En referencia a la ansiedad ante los exámenes, se establecieron tres niveles de ansiedad partiendo de los percentiles $25 \mathrm{y}$ 75. Es decir, en el nivel bajo de ansiedad se englobaron los individuos situados por debajo del percentil 25, en el nivel medio aquellos situados entre el percentil 25 y el 75 , y en el nivel alto los situados por encima del percentil 75. En función de ese criterio para establecer los niveles de ansiedad, el $45.3 \%$ de los parti- cipantes reflejó ansiedad media, y constituyó el grupo más numeroso. Adicionalmente, un $28.9 \%$ de participantes reflejó un nivel alto de ansiedad, mientras que un $25.9 \%$ mostró un nivel bajo.

En cuanto a las relaciones entre las tres variables principales, la Tabla 2 refleja las correlaciones encontradas, entre las que se incluyen las cuatro dimensiones de ansiedad ante los exámenes. 
Tabla 2

Correlaciones entre procrastinación académica, calificación media y ansiedad ante los exámenes.

\begin{tabular}{c|c|c|c|c|c|c|c} 
& PROC & CALIF & ANS & PRE & INTERF & EMOC & F. CONF \\
\hline PROC & - & -.107 & .054 & -.076 & $.335^{* *}$ & .113 & $-.169^{*}$ \\
CALIF & -.107 & - & $-.257^{* *}$ & -.113 & $-.298^{* *}$ & $-.277^{* *}$ & .134 \\
ANS & .054 & $-.257^{* *}$ & - & $.765^{* *}$ & $.523^{* *}$ & $.823^{* *}$ & -.135 \\
PRE & -.076 & -.113 & $.765^{* *}$ & - & $.211^{* *}$ & $.616^{* *}$ & $-.397^{* *}$ \\
INTERF & $.335^{* *}$ & $-.298^{* *}$ & $.523^{* *}$ & $.211^{* *}$ & - & $.355^{* *}$ & $-.348^{* *}$ \\
EMOC & .113 & $-.277^{* *}$ & $.823^{* *}$ & $.616^{* *}$ & $.355^{* *}$ & - & $-.447^{* *}$ \\
F. & $-.169^{*}$ & .134 & -.135 & $-.397^{* *}$ & $-.348^{* *}$ & $-.447^{* *}$ & - \\
CONF & & & & & &
\end{tabular}

Notas: ${ }^{*} p \leq .05 ;{ }^{* *} p \leq .01$. PROC: Procrastinación; CALIF: Calificación media; ANS: Ansiedad global. Dimensiones de ansiedad: PRE: Preocupación; INTERF: Interferencia; EMOC: Emocionalidad; F. CONF: Falta de confianza.

Como se puede observar, existe una relación significativa y negativa entre la ansiedad ante los exámenes y la calificación media. Es decir, mayores niveles de ansiedad ante los exámenes aparecen conectados con resultados académicos que revelan una menor calificación media. Por su lado, las relaciones entre la procrastinación académica y la ansiedad ante los exámenes (teniendo en cuenta la escala global), y entre la procrastinación académica y la calificación media no son significativas.

No obstante, algunas dimensiones concretas de ansiedad sí muestran una relación significativa con la procrastinación. Por un lado, se observa una relación positiva entre la procrastinación y el factor interferencia. Es decir, a más pensamientos intrusivos o irrelevantes que desvían la atención de la tarea, mayor procrastinación. Además, se refleja una relación negativa entre la procrastinación y el factor falta de confianza. Es decir, teniendo en cuenta el sentido inverso de dicho factor, a menor seguridad en la capacidad de uno mismo para realizar la tarea, mayor procrastinación.

Adicionalmente, se ha encontrado relación significativa y negativa entre la calificación media y dos factores concretos de ansiedad ante la evaluación: emocionalidad e interferencia. En primer lugar, a mayor percepción subjetiva de activación fisiológica (emocionalidad), menor rendimiento académico. En segundo lugar, a más pensamientos intrusivos o irrelevantes que desvían la atención de la tarea (interferencia), menor rendimiento académico.

En relación con los análisis de comparación de medias, se obtuvieron los siguientes resultados. En primer lugar, comparando el promedio en la calificación media entre los tres grupos de ansiedad ante los exámenes, el grupo de ansiedad baja es el que obtiene un promedio superior $(M=6.74 ; D T=1.44)$, seguido del grupo de ansiedad media $(M=$ $6.37 ; D T=1.07)$. El grupo de ansiedad alta es el que obtiene una calificación media inferior $(M=5.74 ; D T=1.27)$. Dichas diferencias en la calificación media entre los tres niveles de ansiedad ante los exámenes son significativas $\left(\chi_{(2,201)}^{2}=16.929 ; p=.001\right)$ y pueden observarse en la Figura 1. 


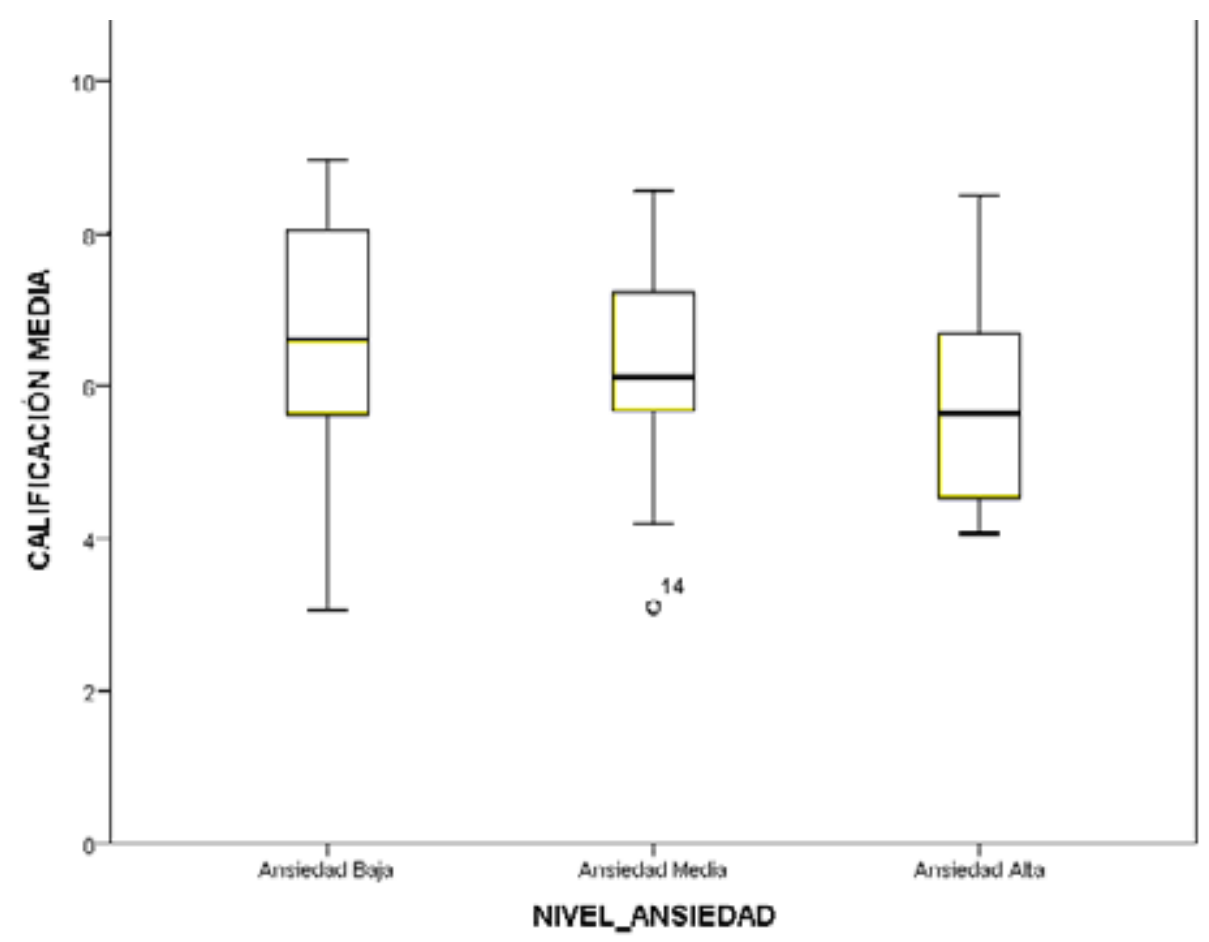

Figura 1. Promedio en calificación media por cada nivel de ansiedad.

Además, comparando los grupos de dos en dos, la diferencia entre el grupo de ansiedad baja y el de ansiedad media no es estadísticamente significativa $(\mathrm{U}=1958.00 ; \mathrm{p}=.087)$. Sin embargo, entre el grupo de ansiedad baja y el de ansiedad alta sí lo es $(\mathrm{U}=895.50 ; \mathrm{p}=$ .001) y entre el grupo de ansiedad media y el de ansiedad alta también $(\mathrm{U}=1819.00 ; \mathrm{p}=.001)$.

Por último, comparando el promedio en la variable procrastinación académica entre los tres grupos de ansiedad ante los exámenes, el grupo de ansiedad baja es el que obtiene un promedio superior $(M=30.75 ; D T=8.00)$ seguido del grupo de ansiedad alta $(M=30.64$; $D T=7.15$ ). El grupo de ansiedad media es el que obtiene la media más baja en procrastinación académica $(M=29.84 ; D T=6.48)$. Sin embargo, estas diferencias no son estadísticamente significativas $\left(\chi_{(2,201)}^{2}=.441 ; p=.802\right)$.

\section{Discusión}

En primer lugar, los datos apoyan la hipótesis de que la ansiedad es un fenómeno presente en población estudiantil, ya que el
$25.9 \%$ de los participantes refleja un nivel bajo de ansiedad, el $45.3 \%$ refleja un nivel medio y el $28.9 \%$ un nivel alto. Los resultados son congruentes con la literatura existente, ya que la ansiedad ante la evaluación se concibe como un fenómeno frecuente en estudiantes (Furlan et al., 2011; Domínguez et al., 2016).

En segundo lugar, los resultados indican que la procrastinación es un fenómeno presente en población estudiantil, ya que la puntuación media obtenida por los participantes en la Escala de Procrastinación Académica (EPA) es de 30.3 puntos, y la puntuación mínima es de 14 puntos. Los resultados coinciden de nuevo con la literatura, ya que la procrastinación se entiende como un fenómeno prevalente, especialmente si se trata de procrastinación académica en población estudiantil (González-Brignardello y Sánchez-Elvira-Paniagua, 2013; Balkis y Duru, 2009).

Por otra parte, la correlación encontrada entre la ansiedad ante las pruebas de evaluación y la calificación media muestra que la ansiedad ante los exámenes se asocia de forma negativa con el rendimiento académico $(r=$ 
$-.257 ; p \leq .01)$. Estos datos coinciden parcialmente con el conocimiento existente, ya que existen investigaciones empíricas que revelan una asociación negativa entre ambas variables (Dordi Nejad et al., 2011; Rana y Mahmood, 2010; Piemontesi y Heredia, 2011). Sin embargo, algunos estudios han encontrado una relación débil o nula en este sentido (Farooqui et al., 2012; Álvarez et al., 2012). En relación con esto último, esta investigación refleja que la ansiedad ante los exámenes se asocia en mayor medida con los resultados académicos si se da en su manifestación cognitiva y/o fisiológica, ya que se han encontrado correlaciones negativas entre la calificación media y los factores interferencia $(r=-.298 ; p \leq .01)$ y emocionalidad $(r=-.277 ; p \leq .01)$, aunque la fuerza de la relación se presenta de forma débil al no alcanzar un mínimo de .30 (Aron y Aron, 2002).

Se rechaza la existencia de una relación negativa entre la procrastinación académica $\mathrm{y}$ el rendimiento académico, ya que no se ha encontrado correlación entre ambos constructos. En este sentido, los resultados no coinciden con la literatura, en la que existen investigaciones que han encontrado correlación entre ambas variables, aunque difieran en la dirección y la fuerza de correlación encontradas. Es decir, algunos estudios han encontrado que la procrastinación se asocia de forma negativa con los resultados académicos (Furlan et al., 2012) y otras investigaciones revelan que la procrastinación académica puede llevar a resultados académicos positivos (Sánchez Hernández, 2010).

Por el contrario, dos factores de ansiedad sí correlacionan de forma significativa con la procrastinación. Se trata de la interferencia $(r$ $=.335 ; p \leq .01)$ y la falta de confianza $(r=$ -.169; $p \leq .05)$. Estos datos confirman parcialmente la hipótesis de que la procrastinación académica se relaciona positivamente con la ansiedad ante los exámenes. Es decir, los resultados obtenidos indican que no hay relación entre la procrastinación académica y la ansiedad ante la evaluación de forma global, pero sí existe asociación entre la procrastinación y dos de las dimensiones de ansiedad. En cuanto al factor interferencia, la correlación es positiva, por tanto, a mayor número de pensamientos intrusivos que interfieren en la atención hacia la tarea, mayor es el nivel de procrastinación. Esto es coherente con la propuesta cognitivo-conductual, que destaca los pensamientos irracionales sobre las implicaciones de la tarea como factores determinantes de la procrastinación (Álvarez-Blas, 2010). Por el contrario, la correlación con el factor falta de confianza es negativa. Teniendo en cuenta el sentido inverso de su denominación, a menor seguridad en la capacidad propia para afrontar la actividad, mayor nivel de procrastinación. Esta última consideración coincide con la literatura, pues diversos estudios indican que las personas procrastinadoras tienen una baja autoeficacia, es decir, una baja confianza en su capacidad de realizar las tareas con éxito (Wolters, 2003; Contreras et al., 2005; Quant y Sánchez, 2012). Además, la asociación de la procrastinación con las dimensiones de ansiedad interferencia y falta de confianza también se encontró en el trabajo de Furlan et al. (2012).

De esta forma, según los resultados de la investigación, se infiere que la procrastinación académica no afecta directamente el rendimiento académico, ya que no se ha encontrado correlación, pero sí afecta de forma indirecta, incidiendo en las manifestaciones cognitivas de la ansiedad (interferencia y falta de confianza), que sí tienen una repercusión directa y negativa sobre el rendimiento académico.

Para terminar, los resultados del análisis de comparación de medias entre los grupos de ansiedad avalan de nuevo la hipótesis de que la ansiedad ante los exámenes se asocia de forma negativa con el rendimiento académico, ya que existen diferencias significativas entre los grupos de ansiedad respecto a la calificación media $\left(\chi_{(2,201)}^{2}=16.929 ; p=.001\right)$. Además, dichas diferencias aparecen solo cuando el grado de ansiedad es alto. Es decir, la 
repercusión negativa en el rendimiento académico solo se produce si el nivel de ansiedad se convierte en elevado. Estos datos coinciden con la literatura existente, que indica que las personas con altos niveles de ansiedad ante la evaluación tienen repercusiones negativas en su rendimiento académico, mientras que las personas con niveles medios de ansiedad usan esa experiencia emocional para prepararse ante las posibles dificultades de la situación de evaluación (Furlan et al., 2014).

En definitiva, los resultados de la presente investigación han puesto de manifiesto la importancia de proponer intervenciones en aquellos factores que repercuten de forma negativa en los resultados académicos. Concretamente, la intervención en la ansiedad ante la evaluación es un aspecto central, ya que tiene un efecto directo sobre el rendimiento académico. De la misma forma, las conductas procrastinadoras son elementos susceptibles de intervención, pues interfieren de forma indirecta en los resultados académicos, asociándose con las manifestaciones cognitivas de la ansiedad ante los exámenes.

El peso relativo de los componentes cognitivos, conductuales, motivacionales y emocionales en la eficacia de las intervenciones sobre la procrastinación y la ansiedad ante los exámenes está aún por determinarse. Sin embargo, los programas que combinan componentes tanto cognitivos como conductuales se muestran más eficaces que otras intervenciones menos complejas (Zeidner, 1998, citado por Furlan, 2013). Además, el efecto positivo de este tipo de intervenciones suele extenderse a otros ámbitos asociados, influyendo positivamente sobre el estado de ánimo general, la percepción de uno mismo y las relaciones interpersonales.

Asimismo, el bajo rendimiento académico es un fenómeno que resulta de múltiples factores, además de la ansiedad ante la evaluación y la procrastinación (Chilca, 2017). Por ello, el presente estudio lanza una propuesta para futuras investigaciones, con el fin de ofrecer intervenciones individualizadas $\mathrm{y}$ eficaces. Se propone indagar en las variables específicas que determinan el bajo rendimiento en alumnos que procrastinan y experimentan ansiedad ante la evaluación, en alumnos que solo muestran una de esas condiciones y en alumnos que no muestran ninguna de ellas, pero aun así obtienen resultados académicos negativos.

De esta forma, se hipotetiza la existencia de ocho categorías de estudiantes, en función de las distintas combinaciones entre los niveles de procrastinación, ansiedad ante los exámenes y rendimiento académico (ver Figura 2). La verificación del modelo debería realizarse a través de un análisis de clusters o clases latentes; sin embargo los datos disponibles en este estudio no permiten realizar dicha comprobación.

- Estudiante deficiente: sería el caso del alumno que muestra altos niveles de procrastinación y de ansiedad ante la evaluación, y que obtiene un bajo rendimiento.

- Estudiante paradójico: el alumno, a pesar de experimentar ansiedad ante los exámenes y procrastinar, mantiene un nivel adecuado de rendimiento.

- Estudiante ansioso ante la evaluación: el alumno no procrastina pero experimenta una alta ansiedad ante la evaluación, y obtiene un bajo rendimiento.

- Estudiante ansioso controlado: el alumno no procrastina, pero muestra elevada ansiedad ante la evaluación. Sin embargo, esa ansiedad no perjudica su rendimiento.

- Estudiante con otras dificultades: el alumno, a pesar de no procrastinar ni experimentar ansiedad, obtiene un bajo rendimiento académico debido a otro tipo de variables.

- Estudiante ideal: el alumno no procrastina ni experimenta ansiedad, y obtiene buenos resultados académicos.

- Procrastinador tradicional: es un alumno que no muestra ansiedad ante la evaluación, pero sí procrastina y obtiene un 
bajo rendimiento.

- Procrastinador controlado: es el que no muestra ansiedad ante la evaluación, pero sí procrastina, y aun así obtiene un buen rendimiento.

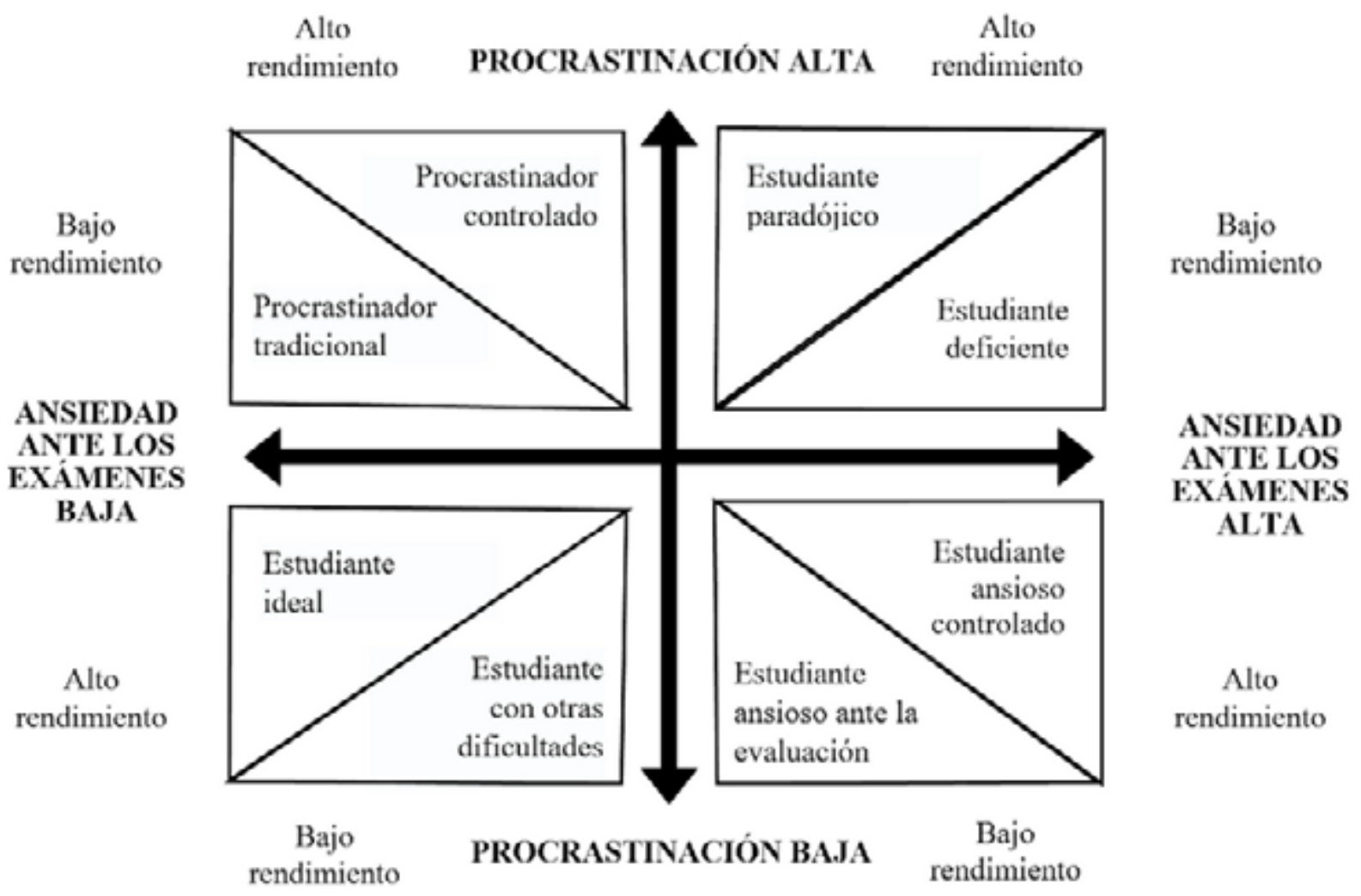

Figura 2. Tipologías de estudiantes según niveles de ansiedad ante los exámenes, procrastinación y rendimiento académico (elaboración propia).

La intervención que se dirija al alumnado debe ser distinta en función de cada perfil de estudiante. De esta forma, si un alumno con bajo rendimiento refleja altos niveles de procrastinación y de ansiedad ante la evaluación, la intervención debería centrarse tanto en la reducción de las conductas de procrastinación como en la gestión de la ansiedad. Sin embargo, si un estudiante muestra un bajo rendimiento sin revelar altos niveles de procrastinación ni de ansiedad, los factores sobre los que se debería intervenir son distintos. En este caso, se podrían evaluar las técnicas de estudio susceptibles de mejora, los procesos cognitivos de atención, la memoria, la concentración, etcétera, que pudieran ser deficientes, así como las situaciones personales que pudieran estar afectando al rendi- miento. Por otro lado, si un estudiante con bajo rendimiento no muestra conductas de procrastinación, pero sí un alto grado de ansiedad ante los exámenes, la intervención debería centrarse de forma específica en el control de la ansiedad en situaciones de evaluación. Igualmente, si un alumno no muestra ansiedad ante la evaluación pero sí realiza conductas de procrastinación que interfieren en su rendimiento, la intervención debería dirigirse de forma específica al aprendizaje de estrategias de planificación, gestión del tiempo y monitorización del estudio.

En relación con el bajo rendimiento académico en la universidad, según el estudio del Instituto Valenciano de Investigaciones Económicas (IVIE), el $33.3 \%$ de los estudiantes que empiezan un grado universi- 
tario no lo termina, ya sea por abandonar la universidad, en el $21.4 \%$ de los casos, o por cambiar de titulación, en el $11.9 \%$. Es decir, partiendo de la información sobre 62 universidades españolas, 48 públicas y 14 privadas, un tercio de los estudiantes abandona el grado universitario que inició, ya sea por dejar definitivamente los estudios en la universidad o por iniciar otro grado (IVIE, 2019).

Sin embargo, los factores que pueden incidir en el abandono de los estudios universitarios no solo se encuentran en el propio sistema educativo. Es decir, existen múltiples variables individuales que pueden perjudicar el rendimiento de un estudiante, aumentando así la probabilidad de que abandone sus estudios. En este sentido, los servicios de atención psicológica y psicopedagógica que ofrecen distintas universidades intervienen en diversos factores que merman el rendimiento académico. Este tipo de servicios trabaja con el alumnado en habilidades como el autocontrol y la relajación, la autoestima, la organización y la gestión del tiempo, etcétera. La adquisición de dichas competencias tiene un efecto preventivo frente a las dificultades académicas. Además, los servicios de atención psicológica y psicopedagógica tienen una función de prevención terciaria ante dichas dificultades, ya que ofrecen asesoramiento psicológico y psicopedagógico individual a aquellos alumnos con problemas en el ámbito personal y/o académico (Saúl, López-González y Bermejo, 2009). Según el trabajo de Hernández y Carranza (2017), el bienestar psicológico es fundamental para lograr un adecuado rendimiento académico, laboral y social. Concretamente, el estudio indica que la felicidad y el optimismo se relacionan de forma positiva con la autorrealización, facilitando así el manejo del estrés y la resolución de problemas. Es decir, si la persona se siente autorrealizada al llevar a cabo sus tareas y responsabilidades, será más fácil que alcance los resultados deseados. Por tanto, el hecho de que los servicios psicológicos universitarios apoyen a los estudiantes en el manejo de ciertas situaciones vitales fomenta un aumento de su bienestar psicológico, mejorando tanto su capacidad de afrontamiento y resolución de problemas como su rendimiento académico.

Para finalizar, se deben resaltar las limitaciones del presente estudio. En primer lugar, se destaca el pequeño tamaño de la muestra $(N=201)$. Asimismo, no se ha considerado la influencia de otras variables de interés como el género, la edad, el curso, el nivel educativo (educación secundaria, bachillerato, educación superior y/o universitaria, entre otros) o el área de conocimiento (ciencias de la salud, ciencias sociales, etcétera), entre otras.

\section{Referencias bibliográficas}

Alegre, A. A. (2016). Relación entre la reflexión en el aprendizaje, la autoeficacia académica y la autorregulación del aprendizaje con la procrastinación académica en estudiantes universitarios de Lima Metropolitana. (Tesis doctoral). Universidad Nacional Mayor de San Marcos, Lima, Perú.

Alegre, A. A. (2013). Autoeficacia y procrastinación académica en estudiantes universitarios de Lima Metropolitana. Propósitos y Representaciones, 1(2), 57-82.

Álvarez, J., Aguilar, J. y Lorenzo, J. J. (2012). La Ansiedad ante los Exámenes en Estudiantes Universitarios: relaciones con variables personales y académicas. Electronic Journal of Research in Educational Psychology, 10(26), 333-354.

Álvarez-Blas, O. R. (2010). Procrastinación general y académica en una muestra de estudiantes de secundaria de Lima metropolitana. Persona, 013, 159-177. https://doi. org/10.26439/persona2010.n013.270

Angarita, D. (2012). Aproximación a un concepto actualizado de la procrastinación. Revista Iberoamericana de Psicología: Ciencia y Tecnología, 5(2), 85-94.

Angarita, D., Sánchez, A. y Barreiro, E. (2012). Relación entre los niveles y tipos de procrastinación académica y el rendimiento académico en estudiantes de psicología de una Univer- 
sidad Privada en Bogotá, Colombia. Memorias del Segundo Congreso Internacional de Psicología y Educación, Universidad Konrad Lorenz, Bogotá, Colombia.

Aron, A. y Aron, E. (2002). Estadística para Psicología. Buenos Aires: Pearson Education.

Balkis, M. y Duru, E. (2009). Prevalence of academic procrastination behavior among pre-service teachers, and its relationship with demographics and individual preferences. Journal of TheoryPractice in Education (JTPE), 5(1), 18-32.

Busko, D. A. (1998).Causes and consequences of perfectionism and procrastination: A structural equation model (Tesis de maestría no publicada). Guelph, Ontario: University of Guelph.

Carranza, R. y Ramírez, A. (2013). Procrastinación y características demográficas asociados en estudiantes universitarios. Apuntes Universitarios. Revista de Investigación, 3(2), 95-108. https://doi.org/10.17162/au.v0i2.284

Cerda, C., Saiz, J. L.y Vergara, D. (2018). Tenacidad en estudiantes universitarios chilenos: un estudio inicial de su estructura y red nomológica. Interdisciplinaria, 35(2), 409-423. https:// doi.org/10.16888/interd.2018.35.2.10

Chilca, M. L. (2017). Autoestima, hábitos de estudio y rendimiento académico en estudiantes universitarios. Propósitos y Representaciones, 5(1), 71-127. https://doi.org/10.20511/ pyr2017.v5n1.145

Contreras, F., Espinosa, J., Esguerra, G., Haikal, A., Polaina, A. y Rodríguez, A. (2005). Autoeficacia, ansiedad y rendimiento académico en adolescentes. Revista Diversitas: Perspectivas en Psicología, 1(2), 183-194. https://doi. org/10.15332/s1794-9998.2005.0002.06

Díaz-Morales, J. F. (2019). Procrastinación: una revisión de su medida y sus correlatos. Revista Iberoamericana de Diagnóstico y Evaluación e Avaliação Psicológica, 51(2), 43-60. https:// doi.org10.21865/RIDEP51.2.04

Domínguez, S. A., Bonifacio, M. y Caro, A. (2016). Prevalencia de ansiedad ante exámenes en una muestra de estudiantes universitarios de Lima Metropolitana. Revista de Psicología, 6(2), 47-56.
Domínguez, S. A., Villegas, G. y Centeno, S. B. (2014). Procrastinación académica: validación de una escala en una muestra de estudiantes de una universidad privada. Liberabit, 20(2), 293-304.

Dordi Nejad, F., Hakimi, H., Ashouri, M., Dehghani, M., Zeinali, Z., Daghighi, M. y Bahrami, N. (2011). On the relationship between test anxiety and academic performance. Procedia, Social and Behavioral Sciences, 15, 3774-3778. https://doi. org/10.1016/j.sbspro.2011.04.372

Farooqui, Y. N., Ghani, R. y Spielberger, C. D. (2012). Gender differences in test anxiety and academic performance of medical students. International Journal of Psychology and Behavioral Sciences, 2(2), 38-43. https://doi. org/10.5923/j.ijpbs.20120202.06

Furlan, L. (2013). Eficacia de una Intervención para Disminuir la Ansiedad frente a los Exámenes en Estudiantes Universitarios Argentinos. Revista Colombiana de Psicología, 22(1), 75-89.

Furlan, L., Ferrero, M. J. y Gallart, G. (2014). Ansiedad ante los exámenes, procrastinación y síntomas mentales en estudiantes de la Universidad Nacional de Córdoba. Revista Argentina de Ciencias del Comportamiento, 6(3), 31-39.

Furlan, L., Heredia, D., Piemontesi, S. y Tuckman, B. (2012). Análisis factorial confirmatorio de la adaptación argentina de la escala de procrastinación de Tuckman (ATPS). Perspectivas en Psicología: Revista de Psicología y Ciencias Afines, 9(3), 142-149.

Furlan, L., Pérez, E., Moyano, M. y Cassady, J. C. (2011). Propiedades psicométricas y estandarización de la Escala de Ansiedad Cognitiva frente a los Exámenes a la población universitaria argentina. Revista Evaluar, 10(1), 22-31.

Gómez, D., Oviedo, R. y Martínez, E. I. (2011). Factores que influyen en el rendimiento académico del estudiante universitario. TECNOCIENCIA Chihuahua, 5(2), 90-97.

González-Brignardello, M. P. y Sánchez-ElviraPaniagua, A. (2013). ¿Puede amortiguar el engagement los efectos nocivos de la procrastinación académica? Acción Psicológica, 10(1), 
117-134. https://doi.org/10.5944/ap.10.1.7039

Heredia, D., Piemontesi, S., Furlan, L. y Volker, H. (2008). Adaptación del Inventario Alemán de Ansiedad frente a los Exámenes: GTAI-A. Revista Evaluar, 8(1), 46-60.

Hernández, R. M. y Carranza, R. F. (2017). Felicidad, optimismo y autorrealización en estudiantes de un programa de educación superior para adultos. Interdisciplinaria, 34(2), 307-325. https://doi.org/10.16888/interd.2017.34.2.5

Hervías, F., Marchena, E. y Menacho, I. (2020). Estimación del pronóstico terapéutico $\mathrm{y}$ análisis del tratamiento en un servicio universitario de psicología. Revista Iberoamericana de Diagnóstico y Evaluación- e Avaliação Psicológica, 54(1), 69-80. https://doi.org/10.21865/ RIDEP54.1.06

Hodapp, V. (1991). The Test Anxiety Inventory TAI-G: An expanded and modified version with fourcomponents. Zeitschriftfür Pädagogische Psychologie, 5, 121-130.

Instituto Valenciano de Investigaciones Económicas (IVIE). (2019). U-Ranking 2019. Indicadores Sintéticos de las Universidades Españolas. https://www.fbbva.es/wp-content/ uploads/2019/04/Informe-U-Ranking-FBBVA-Ivie-2019.pdf

Lamas, H. (2015). Sobre el rendimiento escolar. Propósitos y Representaciones, 3(1), 313-386.

Lay, C. H. (1990). Working to schedule on personal projects: an assessment of person-project characteristics and trait procrastination. Journal of Social Behavior and Personality, 5, 91-103.

Marchena, E., Rapp, C., Hervías, F., Navarro, J. I., Alcalde, C., Aguilar, M. y Menacho, I. (2009). El Servicio de Asesoramiento Psicológico (SAP) de la Universidad de Cádiz. Acción Psicológica, 6(1), 73-85.

Marchena, E., Araujo, A. M., Romero, C., Menacho, I., Hervías, F. y López, M. M. (2011). Participación del servicio de atención psicológica en los espacios de aprendizaje. International Journal of Developmental and Educational Psychology. Revista INFAD, 1(1), 585-602.

Marquina, R. J., Gómez, L., Salas, C., Santibañez, S. y Rumiche, R. (2016). Procrastinación en alumnos universitarios de Lima Metropolitana. Revista Peruana de Obstetricia y Enfermería, 12(1), 11-22.

Montaña, L. A. (2011). Ansiedad en situación de examen y estrategias de afrontamiento en alumnos universitarios de $1^{\circ}$ y $5^{\circ}$ año (Tesina de grado). Facultad de Psicología y Relaciones Humanas, Universidad Abierta Interamericana, Buenos Aires, Argentina.

Moreta, R. y Durán, T. (2018). Propiedades psicométricas de la Escala de Procrastinación Académica (EPA) en estudiantes de psicología de Ambato, Ecuador. Salud y Sociedad, 9(3), 236-247. https://doi.org/10.22199/ S07187475.2018.0003.00003

Pardo, D., Perilla, L. y Salinas, C. (2016). Relación entre procrastinación académica y ansiedad-rasgo en estudiantes de psicología. Cuadernos Hispanoamericanos de Psicología, 14(1), 31-44. https://doi.org/10.18270/ chps..v14i1.1343

Piemontesi, S. y Heredia, D. (2011). Relaciones entre la ansiedad frente a los exámenes, estrategias de afrontamiento, autoeficacia para el aprendizaje autorregulado y rendimiento académico. Revista Tesis, (1), 74-86.

Piemontesi, S., Heredia, D. y Furlan, L. (2012). Propiedades psicométricas de la versión en español revisada del inventario alemán de ansiedad ante exámenes (GTAI-AR) en universitarios argentinos. Universitas Psychologica, 11(1), 177-186. https://doi.org/10.11144/ Javeriana.upsy11-1.ppve

Quant, D. M. y Sánchez, A. (2012). Procrastinación, procrastinación académica: concepto e implicaciones. Revista Vanguardia Psicológica Clínica Teórica y Práctica, 3(1), 45-59.

Rana, R. A. y Mahmood, N. (2010). The relationship between test anxiety and academic achievement. Bulletin of Education and Research, 32(2), 63-74.

Sánchez Hernández, A. M. (2010). Procrastinación académica: un problema en la vida universitaria. Studiositas, 5(2), 87-94.

Saúl, L. A., López-González, M. A. y Bermejo, B. G. (2009). Revisión de los servicios de atención psicológica y psicopedagógica en las 
universidades españolas. Acción Psicológica, 6(1), 17-40. https://doi.org/10.5944/ap.6.1.227 Steel, P. (2007). The nature of procrastination: A meta-analytic and theoretical review of quintessential self-regulatory failure. Psychological Bulletin, 133(1), 65-91. https://doi. org/10.1037/0033-2909.133.1.65

Wolters, C. (2003). Entendimiento de la Procrastinación del self: Regulación de perspectiva. Revista de Educación Psicológica, 95(1), 179-187.

Recibido: 23 de noviembre de 2019 Aceptado: 17 de marzo de 2021 